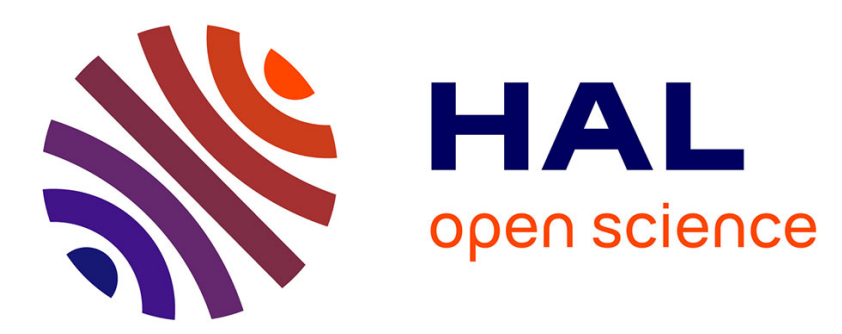

\title{
Optimization of Sign-Preserving Noise-Aided Min-Sum Decoders with Density Evolution
}

F Cochachin, E. Boutillon, D. Declercq

\section{To cite this version:}

F Cochachin, E. Boutillon, D. Declercq. Optimization of Sign-Preserving Noise-Aided Min-Sum Decoders with Density Evolution. International Symposium on Turbo Codes \& Iterative Information Processing 2018 (ISTC'2018), Dec 2018, Hong Kong, China. hal-01950033

\section{HAL Id: hal-01950033 https://hal.science/hal-01950033}

Submitted on 10 Dec 2018

HAL is a multi-disciplinary open access archive for the deposit and dissemination of scientific research documents, whether they are published or not. The documents may come from teaching and research institutions in France or abroad, or from public or private research centers.
L'archive ouverte pluridisciplinaire HAL, est destinée au dépôt et à la diffusion de documents scientifiques de niveau recherche, publiés ou non, émanant des établissements d'enseignement et de recherche français ou étrangers, des laboratoires publics ou privés. 


\title{
Optimization of Sign-Preserving Noise-Aided Min-Sum Decoders with Density Evolution
}

\author{
F. Cochachin*†, E. Boutillon* Senior Member, IEEE and D. Declercq ${ }^{\dagger}$ Senior Member, IEEE \\ *Lab-STICC, UMR 6285, Université de Bretagne Sud, Centre de Recherche BP 92116, Lorient 56321, France \\ ${ }^{\dagger}$ ETIS, ENSEA/University of Cergy-Pontoise/CNRS, 95014 Cergy-Pontoise, France
}

\begin{abstract}
The novelty of this paper is to propose a new LDPC decoder called Sign-Preserving Noise-Aided Min-Sum (SP-NAMS) decoder that improves the decoding performance compared to classical Offset Min-Sum (OMS) decoder when messages are quantized, with only 3 or 4 bits. The particularity of the SPNA-MS decoder is that the variable-to-check messages are never set to 0 , and always carry the sign information. Moreover, the decoder incorporates random perturbation due to deliberate noise injection. The parameters of the SP-NA-MS decoders are optimized in the asymptotic limit of the code length thanks to the Density Evolution (DE) method. The finite-length simulations confirm the conclusions of the DE analysis and gain of up to 0.3 dB in SNR can be obtained compared to regular OMS with same quantization level.
\end{abstract}

\section{INTRODUCTION}

Low-Density Parity-Check (LDPC) codes are widely used in communications standards like DVB-S2, DVB-S2X, IEEE 802.3 [1], etc. LDPC codes can be efficiently decoded by Message-Passing (MP) algorithms that use a Tanner graph [2] representation of the LDPC codes. The Belief-Propagation (BP) decoder has excellent decoding performance in the waterfall region but at a cost of a high computational complexity. The Min-Sum (MS) and Offset Min-Sum (OMS) decoders [3], [4] are simplified version of the BP that are much less complex but at a cost of a slight decoding performance degradation. Reducing the bit-size representation of messages is a technique that further reduces the complexity of the decoder, again, at a cost of performance loss. In the past fifteen years, the quantization of MP decoders has been extensively studied over the Binary-Input Additive White Gaussian Noise (BI-AWGN) channel [4]-[6]. From these works, it is usually advised that 6 bits of quantization gives almost floating point performance. It can also be observed that, to the best authors knowledge, all paper on finite precision use the "no-decision value", i.e., a Log-Likelihood Ratio (LLR) equal to 0 (without defined sign, thus) in their messages representation. This is also true for the recent work on Non-surjective Finite Alphabet Iterative Decoders (NS-FAIDs) [7] which provides a unified framework for several MS-based decoders like Normalized MS (NMS) decoder, OMS decoder, Partially OMS decoder.

In [8], the authors have shown that 3 or 4 bits quantized decoder can benefit from the introduction of particular randomness applied in the amplitude of messages during the decoding process. Thanks to the Density Evolution (DE) method [9], optimized parameters of noise injection could be determined in the asymptotic mode. Those type of decoders are called Noise-Against-Noise MS (NAN-MS) decoders. In this paper, we propose a new type of decoder for low complexity hardware implementation (i.e. 3 or 4 bits only for finite length representation of messages) that always preserve the sign of the messages. In other words, we forbid the 0 value in the message alphabet during the iterative decoding, i.e. a message cannot be erased. In order to optimize this new decoder, we use the optimization method proposed in [8], i.e. we introduce a certain level of randomness in the optimization process. The result can be either a decoder that keeps some randomness, named Sign-Preserving NoiseAided Min-Sum (SP-NA-MS) decoder, or a deterministic decoder that combines MS and OMS behaviors, named SignPreserving decoder.

DE allows us to show that a 4-bits SP-NA-MS decoder can have the same DE threshold than a classical 5-bit OMS decoder. Our DE analysis is corroborated by finite length Monte Carlo simulations of various regular LDPC codes. Those results open new possibilities for massive parallel implementation of LDPC decoders where in average, 100 to 1000 bits are decoded per clock cycle, allowing $100 \mathrm{Gbit} / \mathrm{s}$ up to $1 \mathrm{Tbit} / \mathrm{s}$ decoders required in optical fiber applications.

Due to paper size limitation, in this paper we present only the results for regular LDPC codes, but the proposed decoder can be used to decode irregular LDPC codes. The outline of the paper is as follows. Section II introduces the basic notions of quantized classical decoders and LDPC codes. In Section III, we explain how to preserve the sign of the exchanged messages. In Section IV, we discuss the probabilistic error model used to inject noise to the quantized decoders, and briefly explain how to optimize the model parameters with DE. In section $\mathrm{V}$, we present the results of the asymptotic analysis of SP-NA-MS decoders for regular LDPC codes. Section VI shows finite length performance validation of the gains obtained with the proposed SP-NA-MS decoders, and section VII concludes the paper.

\section{BASIC Notions of ClassicAl QuANTIZED DECODERS AND LDPC CODES}

An LDPC code is a linear block code defined by a sparse parity-check matrix $H=\left[h_{m n}\right]$ of $M$ rows by $N$ columns, with $M<N$. The usual graphical representation of an LDPC code is made by a Tanner graph which is a bipartite graph $G$ composed of two types of nodes, the variable nodes (VNs) $v_{n}, n=1 \ldots N$ and the check nodes $(\mathrm{CNs}) c_{m}, m=1 \ldots M$. A 
VN in the Tanner graph corresponds to a column of $H$ and a $\mathrm{CN}$ corresponds to a row of $H$, with an edge connecting $\mathrm{CN}$ $c_{m}$ to VN $v_{n}$ exists if and only if $h_{m n} \neq 0$. Let us assume that $v$ is any $\mathrm{VN}$ and $c$ is any $\mathrm{CN}$. Let us also denote $\mathcal{V}(v)$ the set of neighbors of a $\mathrm{VN} v$, and denote $\mathcal{V}(c)$ the set of neighbors of a $\mathrm{CN} c$. The degree of a node is the number of its neighbors in $G$. A code is said to have a regular columnweight $d_{v}=|\mathcal{V}(v)|$ if all VNs $v$ have the same degree $d_{v}$. Similarly, if all CNs $c$ have the same degree $d_{c}=|\mathcal{V}(c)|$, a code is said to have a regular row-weight $d_{c}$.

Let $\boldsymbol{x}=\left(x_{1}, \ldots, x_{N}\right) \in\{0,1\}^{N}$ denote a codeword which satisfies $H \boldsymbol{x}^{T}=0$. In this paper, $\boldsymbol{x}$ is mapped by the BPSK modulation and transmitted over the BI-AWGN channel with noise variance $\sigma^{2}$. The channel output $\boldsymbol{y}=\left(y_{1}, \ldots, y_{N}\right)$ is modeled by $y_{n}=\left(1-2 x_{n}\right)+z_{n}$ for $n=1, \ldots, N$, where $z_{n}$ is a sequence of independent and identically distributed (i.i.d.) Gaussian random variables with zero mean and variance $\sigma^{2}$. For quantized decoders the message alphabet $\mathcal{A}_{C}$ is defined as $\mathcal{A}_{C}=\left\{-N_{q}, \ldots,-1,0,+1, \ldots,+N_{q}\right\}$ consists of $N_{s}=2 N_{q}+$ 1 states, with $N_{q}=2^{(q-1)}-1$ and where $q$ is the number of quantization bits. Let us denote $\mathcal{A}_{L}$ the decoder input alphabet, and denote $\ell \in \mathbb{N}$ the number of iterations. Let us also denote $m_{v \rightarrow c}^{(\ell)} \in \mathcal{A}_{L}$ (resp. $m_{c \rightarrow v}^{(\ell)} \in \mathcal{A}_{L}$ ) the message sent from VN $v$ to $\mathrm{CN} c$ (resp. $\mathrm{CN} c$ to $\mathrm{VN} v$ ) in the $\ell^{\text {th }}$ iteration. The LLR can be computed at the channel output as

$$
\operatorname{LLR}\left(y_{n}\right)=\log \left(\frac{\operatorname{Pr}\left(y_{n} \mid x_{n}=0\right)}{\operatorname{Pr}\left(y_{n} \mid x_{n}=1\right)}\right)=\frac{2 y_{n}}{\sigma^{2}} .
$$

We assume in this paper that $\mathcal{A}_{C}=\mathcal{A}_{L}$, hence, $\operatorname{LLR}\left(y_{n}\right)$ has to be quantized and saturated. Let us denote the quantizer by $\mathcal{Q}: \mathbb{R} \rightarrow \mathcal{A}_{L}$, defined as

$$
\mathcal{Q}(y)=\mathcal{S}\left(\lfloor\alpha \times y+0.5\rfloor, N_{q}\right),
$$

where $\lfloor$.$\rfloor depicts the floor function and \mathcal{S}\left(x, N_{q}\right)$ is the saturation function clipping the value of $x$ in the interval $\left[-N_{q}, N_{q}\right]$, i.e. $\mathcal{S}\left(x, N_{q}\right)=\min \left(\max \left(x,-N_{q}\right),+N_{q}\right)$. The parameter $\alpha$ is called channel gain factor and is used to enlarge or decrease the standard deviation of quantized values at the decoder input, $\alpha$ can be seen as an extra degree of freedom in the quantized decoder definition that can be analyzed and optimized on the BI-AWGN channel. With those notations, we define the quantized version of the LLR that initialize the MP decoder by the vector $\boldsymbol{I}=\left(I_{1}, \ldots, I_{N}\right) \in \mathcal{A}_{C}^{N}$, with

$$
I_{n}=\mathcal{Q}\left(L L R\left(y_{n}\right)\right) \quad \forall n=1, \ldots, N .
$$

A MP decoder exchanges messages between $\mathrm{VNs}$ and $\mathrm{CNs}$ along edges using a Tanner graph. Let us briefly recall the VN update (VNU) and $\mathrm{CN}$ update (CNU) equations for classical MS-based decoders. The update rule at a CNU is given by

$m_{c_{m} \rightarrow v_{n}}^{(\ell)}=\left(\prod_{v \in \mathcal{V}\left(c_{m}\right) \backslash\left\{v_{n}\right\}} \operatorname{sign}\left(m_{v \rightarrow c_{m}}^{(\ell)}\right)\right)\left(\min _{v \in \mathcal{V}\left(c_{m}\right) \backslash\left\{v_{n}\right\}}\left(\left|m_{v \rightarrow c_{m}}^{(\ell)}\right|\right)\right)$.

The update rule at a VNU is expressed as

$$
m_{v_{n} \rightarrow c_{m}}^{(\ell+1)}=\Lambda\left(I_{n}+\sum_{c \in \mathcal{V}\left(v_{n}\right) \backslash\left\{c_{m}\right\}} m_{c \rightarrow v_{n}}^{(\ell)}\right),
$$

Table I: Binary representation of the quantized values.

\begin{tabular}{|c|c||c|c|c|}
\hline \multicolumn{2}{|c||}{ Classical Decoder } & \multicolumn{3}{c|}{ SP-NA-MS Decoder } \\
\hline \hline$m \in \mathcal{A}_{C}$ & $q=3$ bits & $m \in \mathcal{A}_{S}$ & $q=3$ bits & $(\operatorname{sign}(m),|m|)$ \\
\hline-3 & 101 & -3 & 111 & $(-1,3)$ \\
\hline-2 & 110 & -2 & 110 & $(-1,2)$ \\
\hline-1 & 111 & -1 & 101 & $(-1,1)$ \\
\hline- & 100 & -0 & 100 & $(-1,0)$ \\
\hline 0 & 000 & +0 & 000 & $(+1,0)$ \\
\hline+1 & 001 & +1 & 001 & $(+1,1)$ \\
\hline+2 & 010 & +2 & 010 & $(+1,2)$ \\
\hline+3 & 011 & +3 & 011 & $(+1,3)$ \\
\hline
\end{tabular}

where $\left.\Lambda(x)=\operatorname{sign}(x) \times \mathcal{S}\left(\max \left(|x|-\lambda_{v}, 0\right)\right), N_{q}\right)^{1}$. We define the classical OMS with offset value $\lambda_{v} \neq 0$, where the special case of $\lambda_{v}=0$ corresponds to the classical MS.

\section{Sign-Preserving Noise-Aided Min-Sum DECODERS}

In the classical MS-based decoders, the value of the v-to-c message can be zero, see (5). In that case, the erased message, i.e. $m_{v_{n} \rightarrow c_{m}}^{(\ell+1)}=0$, does not carry any information and does not participate in the convergence of the decoder. In this paper, we propose a new type of decoder, with a modified VNU using a sign preserving factor, which never propagates erased messages.

\section{A. Quantization used for SP-NA-MS Decoders}

Using the sign-and-magnitude representation one can obtain a message alphabet which is symmetric around zero and which is composed of $2^{q}$ states. Hence the message alphabet for SP-NA-MS decoders denoted by $\mathcal{A}_{S}$ is defined as $\mathcal{A}_{S}=$ $\left\{-N_{q}, \ldots,-1,-0,+0,+1, \ldots,+N_{q}\right\}^{2}$. The sign of a message $m \in \mathcal{A}_{S}$ indicates the estimated bit value associated with the $\mathrm{VN}$ to or from which $m$ is being passed while the magnitude $|m|$ of $m$ represents its reliability. In this paper, it is assumed that $\mathcal{A}_{L}=\mathcal{A}_{S}$. An example of the binary representation of $\mathcal{A}_{C}$ and $\mathcal{A}_{S}$ for $q=3$ is shown in Table I, one can see that -0 is represented by $100_{2},+0$ is represented by $000_{2}$, etc. The quantization process defined in (2) is replaced by

$$
\mathcal{Q}^{*}(y)=\left(\operatorname{sign}(y), \mathcal{S}\left(\lceil\alpha \times|y|\rceil-1, N_{q}\right)\right),
$$

where $\lceil$.$\rceil depicts the ceiling function. The quantized LLR is$ thus defined as $I_{n}=\mathcal{Q}^{*}\left(\operatorname{LLR}\left(y_{n}\right)\right) \in \mathcal{A}_{S}$ for $n=1, \ldots, N$. Let us define the update rules for Sign-Preserving decoders.

\section{B. Sign-Preserving Min-Sum Decoders}

One can note from (4) that the CNU, by construction, generates outgoing messages that always belong to $\mathcal{A}_{S}$. In the case of the VNU, (5) should be modified to ensure that the outgoing message will always belong to $\mathcal{A}_{S}$. To preserve always the sign of the messages, let us denote by $\mu_{v_{n} \rightarrow c_{m}}^{(\ell)}$ the sign-preserving factor of the message $m_{v_{n} \rightarrow c_{m}}^{(\ell+1)}$, defined as

$$
\mu_{v_{n} \rightarrow c_{m}}^{(\ell)}=\xi \times \operatorname{sign}\left(I_{n}\right)+\sum_{c \in \mathcal{V}\left(v_{n}\right) \backslash\left\{c_{m}\right\}} \operatorname{sign}\left(m_{c \rightarrow v_{n}}^{(\ell)}\right),
$$

\footnotetext{
${ }^{1}$ Note that in the literature $\lambda_{v}$ is often applied at the $\mathrm{CN}$. Applying the offset at the $\mathrm{VN}$ or at the $\mathrm{CN}$ is equivalent only when the saturation function is not used since $\min _{i=1, \ldots, n}\left(\left|x_{i}\right|-\lambda_{v}\right)=\min _{i=1, \ldots, n}\left(\left|x_{i}\right|\right)-\lambda_{v}$.

${ }^{2}$ The alphabet $\mathcal{A}_{S}$ can be easily implemented in hardware because each value of $\mathcal{A}_{S}$ has its corresponding binary representation.
} 
where $\xi=1+\left(\left(d_{v}+1\right) \bmod 2\right)$ where $d_{v}$ is the degree of the $\mathrm{VN} v$. Note that the other values of $\xi$ give worse decoding performance.

From (7), one can note that, by construction, $\mu_{v_{n} \rightarrow c_{m}}^{(\ell)}$ is the sum of $d_{v}$ (resp. $d_{v}+1$ ) values in $\{-1,+1\}$ if $d_{v}$ is odd (resp. if $d_{v}$ is even). Thus, $\mu_{v_{n} \rightarrow c_{m}}^{(\ell)}$ is always an odd number.

The update rule of the Sign-Preserving Offset Min-Sum (SPOMS) VNU is changed from (5) to

$$
m_{v_{n} \rightarrow c_{m}}^{(\ell+1)}=\Lambda^{*}\left(\frac{\mu_{v_{n} \rightarrow c_{m}}^{(\ell)}}{2}+I_{n}+\sum_{c \in \mathcal{V}\left(v_{n}\right) \backslash\left\{c_{m}\right\}} m_{c \rightarrow v_{n}}^{(\ell)}\right) \text {, }
$$

where $\Lambda^{*}(x)=\left(\operatorname{sign}(x), \mathcal{S}\left(\max \left(\lfloor|x|\rfloor-\lambda_{v}, 0\right), N_{q}\right)\right)$. Note that $\Lambda^{*}($.$) is applied on a non-null value since by construction,$ the fractional part of $\left(\mu_{v_{n} \rightarrow c_{m}}^{(\ell)}\right) / 2$ is 0.5 .

We define a Sign-Preserving Min-Sum (SP-MS) with $\lambda_{v}=0$.

\section{Sign-Preserving Noise-Aided Min-Sum Decoders}

The noisy version of the SP-MS decoder, i.e., the SP-NAMS decoder is defined by introduction some randomness during the VNU processing following the same principle as for the NAN-MS decoder of [8]. The SP-NA-MS decoder is implemented perturbing unsaturated v-to-c messages $\hat{m}_{v_{n} \rightarrow c_{m}}^{(\ell+1)}$. In (8), the saturation function $\mathcal{S}$ in the function $\Lambda^{*}$ is not used to compute $\hat{m}_{v_{n} \rightarrow c_{m}}^{(\ell+1)}$, i.e. we use $\Lambda^{*}(x)=(\operatorname{sign}(x),\lfloor|x|\rfloor)$.

The update rule for a noisy-VNU is given by

$$
\tilde{m}_{v_{n} \rightarrow c_{m}}^{(\ell+1)}=\Upsilon\left(\hat{m}_{v_{n} \rightarrow c_{m}}^{(\ell+1)}\right),
$$

where $\Upsilon$ is a noise model which is defined in the next section. Note that the $\Upsilon$ performs also the saturation operation.

\section{Probabilistic Error Model and Noisy Density EVOLUTION FOR SP-NA-MS DECODERS}

In this section, we first introduce the constraints on the noise models, then we present the noise model that we use to perturb $\hat{m}$. Finally, we briefly explain how to optimize the model parameters with noisy DE.

\section{A. Probabilistic Error Model for SP-NA-MS Decoders}

DE analysis can be performed only using memoryless noise models which must satisfy the following condition of symmetry [8]

$$
\operatorname{Pr}\left(\Upsilon\left(\beta_{1}\right)=\beta_{2}\right)=\operatorname{Pr}\left(\Upsilon\left(-\beta_{1}\right)=-\beta_{2}\right), \quad \forall \beta_{1}, \beta_{2},
$$

these noise models allow the VNUs to be symmetric, allowing to use the all-zero codeword assumption necessary in DE [9]. Moreover, since the addition of noise is independent of the sign of the message, we will suppose in the sequel without loss of generality that the message $\hat{m}$ is always positive.

Let us denote $\mathcal{A}_{N}$ the alphabet of $\hat{m}$, with $\mathcal{A}_{N}=\left\{-N_{q} \times\right.$ $\left.\left.d_{v}-\left\lfloor d_{v} / 2\right\rfloor\right), \ldots,-0,+0, \ldots,+N_{q} \times d_{v}+\left\lfloor d_{v} / 2\right\rfloor\right\}$. The function $\Upsilon$ is defined as $\Upsilon: \mathcal{A}_{N} \rightarrow \tilde{\mathcal{A}}_{S}$, where $\tilde{\mathcal{A}}_{S}=\mathcal{A}_{S}$, with the random process that transforms $\hat{m} \in \mathcal{A}_{N}$ into $\tilde{m} \in \tilde{\mathcal{A}}_{S}$ defined by the conditional probability density function (CPDF) $\operatorname{Pr}(\Upsilon(\hat{m})=\tilde{m}) . \Upsilon$ is parametrized by three different transition
Table II: Conditional probability density function of $\Upsilon$.

\begin{tabular}{|c|c|c|c|c|c|}
\hline$\tilde{m} \backslash \hat{m}$ & +0 & +1 & $+1<.<+N_{q}$ & $+N_{q}$ &.$>+N_{q}$ \\
\hline$\tilde{m}=\hat{m}-1$ & 0 & $\varphi_{0}$ & $\varphi_{a}$ & $\varphi_{s}$ & 0 \\
\hline$\tilde{m}=\hat{m}$ & 1 & $1-\varphi_{0}$ & $1-\varphi_{a}$ & $1-\varphi_{s}$ & 0 \\
\hline$\tilde{m}=N_{q}$ & 0 & 0 & 0 & 0 & 1 \\
\hline
\end{tabular}

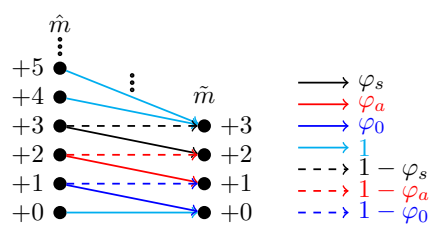

Figure 1: The mapping used for the noise model $\Upsilon$.

probabilities, denoted $\varphi=\left(\varphi_{s}, \varphi_{a}, \varphi_{0}\right) \cdot \varphi_{s}$ denotes the CPDF $\operatorname{Pr}\left(\tilde{m}=+N_{q}-1 \mid \hat{m}=+N_{q}\right), \varphi_{a}$ denotes the CPDF $\operatorname{Pr}(\tilde{m}=$ $\beta-1 \mid \hat{m}=\beta), \forall \beta \in\left\{+2, \ldots,+\left(N_{q}-1\right)\right\}$, and finally $\varphi_{0}$ denotes the $\mathrm{CPDF} \operatorname{Pr}(\tilde{m}=+0 \mid \hat{m}=+1)$. The saturation function is performed by setting $\operatorname{Pr}\left(\tilde{m}=+N_{q} \mid \hat{m}>+N_{q}\right)=$ 1. Table II give the resulting CPDF.

The rationale behind $\Upsilon$ is to implement a probabilistic offset with the purpose of always keeping the sign of the messages. Let us discuss the case of $\varphi_{s}=\varphi_{a}=\varphi_{0}$. It can see that SPMS and SP-OMS decoders are implemented with $\varphi=(0,0,0)$ and $\boldsymbol{\varphi}=(1,1,1)$, respectively. For other values of $\boldsymbol{\varphi}$, the offset is only applied from time to time during the decoding iterations, implementing a probabilistic weighted combination of a SP-MS decoder and a SP-OMS decoder.

With $\varphi_{s}$ and $\varphi_{0}$, the extreme values of $\mathcal{A}_{S}$ are studied. $\hat{m}=$ \pm 1 is a special case because it will be changed to $\tilde{m}= \pm 0$ with probability $\varphi_{0}$. Since $\tilde{m}= \pm 0$ propagates the sign but with a reliability of zero, therefore, $\varphi_{0}$ has to be analyzed differently than $\varphi_{a}$. Additionally, in quantized decoders, all values greater than $N_{q}$ are saturated to $N_{q}$. As a result, many more configurations of the VNU states lead to $\tilde{m}_{v_{n} \rightarrow c_{m}}^{(\ell)}=N_{q}$ compared to other values, and $\varphi_{s}$ should also be analyzed differently than $\varphi_{a}$. Fig. 1 depicts $\Upsilon$ for $\left(q=3, N_{q}=3\right)$.

\section{B. Noisy Density Evolution for SP-NA-MS Decoders}

The goal of DE is to recursively compute the probability mass function (PMF) of the exchanged messages in the Tanner graph along the iterations. DE allows us to predict if an ensemble of LDPC codes, parametrized by its degree distribution, decoded with a given MP decoder, converges to zero error probability in the limit of infinite block length. For the BI-AWGN channel, the maximum value of $\sigma$ or the minimum SNR at which the DE converges to a zero error probability is called the DE threshold $\delta$, as in [8] $\delta_{d b}=10 \log _{10}\left(\frac{1}{2 R \sigma^{* 2}}\right)$ where $\sigma^{*}=\delta$, and $R$ is the rate of the code.

In this paper the details of the noisy-DE equations are not presented, we refer to [10], [11] for more details. The noisyDE threshold $\tilde{\delta}$ is a function of the code family, parametrized by its degree distribution $\left(d_{v}, d_{c}\right)$, of the number of precision bits $q$, of the value of the channel gain factor $\alpha$, and of the values of the transition probabilities of the noise model 
$\left(\varphi_{s}, \varphi_{a}, \varphi_{0}\right)$. For a fixed $q$ and a fixed $\left(d_{v}, d_{c}\right), \tilde{\delta}$ is used to jointly optimize $\left(\varphi_{s}, \varphi_{a}, \varphi_{0}\right)$ and $\alpha$ :

$$
\left(\varphi_{s}^{*}, \varphi_{a}^{*}, \varphi_{0}^{*}, \alpha^{*}\right)=\arg \max _{\left(\varphi_{s}, \varphi_{a}, \varphi_{0}, \alpha\right)}\left\{\tilde{\delta}\left(d_{v}, d_{c}, q, \alpha,\left(\varphi_{s}, \varphi_{a}, \varphi_{0}\right)\right)\right\} .
$$

\section{ASYMPTOTIC ANALYSIS FOR REgular LDPC CODES}

In this section, we consider the ensemble of $\left(d_{v}, d_{c}\right)$-regular LDPC codes with code rate $R \in\{1 / 2,3 / 4\}$ for $d_{v} \in\{3,4\}$, $R=0.8413$ for the IEEE 802.3 ETHERNET code [1], and quantized decoders with $q \in\{3,4\}$. The optimization of the transition probabilities of the noise model $\Upsilon$ and the channel gain factor is made using a greedy algorithm which computes a local maximum DE threshold. For noiseless classical decoders, the optimization (10) is reduced to the optimum channel gain factor $\alpha^{*}$ which is computed performing a grid-search.

The DE thresholds of the noiseless classical MS and OMS decoders are given in Table III. It can be seen that the OMS is almost always superior to the MS for the considered cases, which was expected, except for the regular $d_{v}=3 \mathrm{LDPC}$ codes with low precision $q=3$.

Table III: DE thresholds of classical MS and OMS decoders.

\begin{tabular}{|c|c|c|c|c|c|c|c|}
\hline \multicolumn{8}{|c|}{$\left(d_{v}, d_{c}\right)$-regular LDPC code, BI-AWGN channel } \\
\hline & & \multicolumn{2}{|c|}{$\left(d_{v}=3, d_{c}=6\right)$} & \multicolumn{2}{|c|}{$\overline{\left(d_{v}=3, d_{c}=12\right)}$} & \multicolumn{2}{|c|}{$\overline{\left(d_{v}=6, d_{c}=32\right)}$} \\
\hline$q$ & $\lambda_{v}$ & $\alpha^{*}$ & $\delta_{d b}$ & $\alpha^{*}$ & $\delta_{d b}$ & $\alpha^{*}$ & $\delta_{d b}$ \\
\hline \multirow{2}{*}{3 bits } & 0 & 0.9375 & 1.7888 & 0.625 & 2.7316 & 0.455 & 4.0812 \\
\hline & 1 & 1.0625 & 2.2039 & 0.9375 & 3.1343 & 0.84 & 3.5928 \\
\hline \multirow{2}{*}{4 bits } & 0 & 2.0 & 1.6437 & 1.25 & 2.5646 & 1.035 & 3.8154 \\
\hline & 1 & 1.875 & 1.3481 & 1.5 & 2.4484 & 1.28 & 3.1685 \\
\hline \multirow{2}{*}{5 bits } & 0 & 4.0 & 1.6132 & 2.5 & 2.5268 & 1.985 & 3.7506 \\
\hline & 1 & 2.625 & 1.2154 & 2.25 & 2.3040 & 1.45 & 3.1400 \\
\hline & & \multicolumn{2}{|c|}{$\left(d_{v}=4, d_{c}=8\right)$} & \multicolumn{2}{|c|}{$\left(d_{v}=4, d_{c}=16\right)$} & & \\
\hline$q$ & $\lambda_{v}$ & $\alpha^{*}$ & $\delta_{d b}$ & $\alpha^{*}$ & $\delta_{d b}$ & & \\
\hline \multirow{2}{*}{3 bits } & 0 & 0.8125 & 2.7360 & 0.6875 & 3.1550 & & \\
\hline & 1 & 1.25 & 2.3219 & 0.9375 & 3.0632 & & \\
\hline \multirow{2}{*}{4 bits } & 0 & 1.625 & 2.5389 & 1.375 & 2.9441 & & \\
\hline & 1 & 1.75 & 1.7509 & 1.5 & 2.5292 & & \\
\hline \multirow{2}{*}{5 bits } & 0 & 3.25 & 2.4948 & 2.75 & 2.8991 & & \\
\hline & 1 & 2.0 & 1.7061 & 1.875 & 2.4606 & & \\
\hline
\end{tabular}

In Table IV, we indicate the noisy DE thresholds obtained with (10), we also show the DE gains obtained comparing the best thresholds indicated in bold in Table III and the noisy thresholds of SP-NA-MS decoders. Moreover, we list the best noisy DE thresholds of NAN-MS decoders presented in [8]. Several conclusions can be derived from this analysis.

1) First, the DE thresholds of the SP-NA-MS decoders are almost always better than the DE thresholds of the noiseless classical decoders. The DE gains for the SP-NA-MS decoders are quite important for $q=3$, the largest gain obtained is around $0.3399 \mathrm{~dB}$ for $\left(d_{v}=4, d_{c}=8\right)$. While the DE gains are smaller for the largest precision $q=4$. We can observe a loss of around $0.0102 \mathrm{~dB}$ for $\left(d_{v}=6, d_{c}=32\right)$ and $q=4$. From this analysis, we can conclude that the preservation of the sign of messages and the noise injection are more and more beneficial as the decoders are implemented in low precision. 2) Second, when comparing the noisy thresholds of SP-NAMS and NAN-MS decoders, one can observe that the SPNA-MS decoders achieve better DE thresholds for almost all $\left(d_{v}, d_{c}\right)$-regular LDPC codes tested, the only exception appears for the regular $\left(d_{v}=6, d_{c}=32\right)$ LDPC code and $q=4$. The largest gain obtained, when comparing the SP-NAMS thresholds and NAN-MS thresholds, is around $0.1803 \mathrm{~dB}$ for the regular $\left(d_{v}=6, d_{c}=32\right)$ LDPC code and $q=3$.

3) A third remark comes from the interpretation of the optimum $\varphi^{*}$ obtained through the DE analysis. We have $\varphi_{0}^{*}=0$ for regular $d_{v}=3$ LDPC codes, this makes sense because $d_{v}=3$ is small enough to transform $\hat{m}= \pm 1$ into $\tilde{m}= \pm 0$, which gives to $\tilde{m}_{v_{n} \rightarrow c_{m}}$ a reliability of zero and which could not help to extrinsic messages become more and more reliable at each new decoding iteration. For regular $d_{v}>3$ LDPC codes, we have almost always $\varphi_{0}^{*}=1$, hence, one can conclude that for regular $d_{v}>3$ LDPC codes, the transformation from $\hat{m}= \pm 1$ to $\tilde{m}= \pm 0$, does not affect the decoding process. Note that in [8], the transformation from $\hat{m}= \pm 1$ to $\tilde{m}=0$ should not be allowed since $\tilde{m}=0 \in \mathcal{A}_{C}$ erase the bit value.

4) Finally, all SP-NA-MS decoders can be implemented as deterministic decoders since the values of the transition probabilities are close or equal to 0 or 1 . The optimum noise parameters $\varphi^{*}$ are close to $\left(\varphi_{s}^{*}, \varphi_{a}^{*}, \varphi_{0}^{*}\right)=(1,1,0)$ for the regular $d_{v}=3$ LDPC codes. While in the case of the regular $d_{v}>3$ LDPC codes, $\varphi^{*}$ are close to $\left(\varphi_{s}^{*}, \varphi_{a}^{*}, \varphi_{0}^{*}\right)=(1,1,1)$ which correspond to a deterministic SP-OMS decoder.

Table IV: Noisy DE thresholds of SP-NA-MS decoders

\begin{tabular}{|c|c|c|c|c|c|c|c|c|}
\hline \multicolumn{8}{|c|}{ SP-NA-MS decoders, $\left(d_{v}, d_{c}\right)$-regular LDPC code, BI-AWGN channel } & NAN-MS \\
\hline$\left(d_{v}, d_{c}\right)$ & 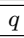 & $\bar{\alpha}^{*}$ & $\varphi_{s}^{*}$ & $\varphi_{a}^{*}$ & $\varphi_{0}^{*}$ & $\tilde{\delta}_{d b}$ & DE gain & $\tilde{\delta}_{d b}-\mathrm{NIV}$ \\
\hline \multirow{2}{*}{$(3,6)$} & 3 & 0.96 & 0.987 & 0.712 & 0.000 & 1.4994 & 0.2894 & 1.5711 \\
\hline & 4 & 1.79 & 1.000 & 1.000 & 0.000 & 1.2688 & 0.0793 & 1.2877 \\
\hline \multirow{2}{*}{$(3,12)$} & 3 & 0.72 & 1.000 & 0.725 & 0.000 & 2.5421 & 0.1895 & 2.5989 \\
\hline & 4 & 1.34 & 1.000 & 0.938 & 0.000 & 2.3596 & 0.0888 & 2.3777 \\
\hline \multirow{2}{*}{$(4,8)$} & 3 & 1.01 & 0.900 & 1.000 & 1.000 & 1.9820 & 0.3399 & 2.1056 \\
\hline & 4 & 1.54 & 1.000 & 1.000 & 1.000 & 1.7306 & 0.0203 & 1.7411 \\
\hline \multirow{2}{*}{$(4,16)$} & 3 & 0.75 & 1.000 & 0.962 & 0.712 & 2.7448 & 0.3184 & 2.8121 \\
\hline & 4 & 1.30 & 1.000 & 1.000 & 1.000 & 2.4941 & 0.0351 & 2.5077 \\
\hline \multirow{2}{*}{$(6,32)$} & 3 & 0.74 & 1.000 & 1.000 & 1.000 & 3.3963 & 0.1965 & 3.5766 \\
\hline & 4 & 1.18 & 1.000 & 1.000 & 1.000 & 3.1787 & -0.0102 & 3.1685 \\
\hline
\end{tabular}

\section{Finite Length Performance}

In order to corroborate the asymptotic results obtained in the previous section, the frame error rate (FER) performance of quantized decoders over the BI-AWGN channel is presented. For this purpose, we designed four $\left(d_{v}, d_{c}\right)$-regular QC-LDPC codes with length $N=1296, R \in\{1 / 2,3 / 4\}$ for $d_{v} \in\{3,4\}$, and circulant size $L=54$ for $R=1 / 2$ and $L=27$ for $R=$ $3 / 4$, using the PEG algorithm from [12]. The classical OMS decoder performance for $q=5$ is also shown as benchmark. A maximum of 100 iterations has been set for $d_{v}=3$ and $d_{v}=4$ LDPC decoders. Fig. 2 shows the FER performance comparisons between the classical MS, classical OMS, and SP-NA-MS decoders, for the two considered precisions $q=$ 3 and $q=4$, and for the regular $\left(d_{v}=3, d_{c}=6\right)$ QCLDPC code. Fig. 3 draws the same curves for the regular $\left(d_{v}=4, d_{c}=8\right)$ QC-LDPC code. A first conclusion is that the finite length FER performance are in accordance with the gains predicted by the DE analysis. We observe in the waterfall 


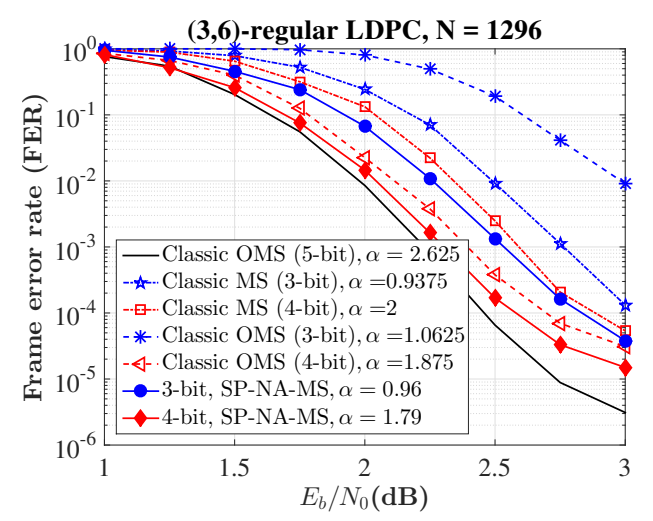

Figure 2: FER performance for $(3,6)$-regular LDPC code.

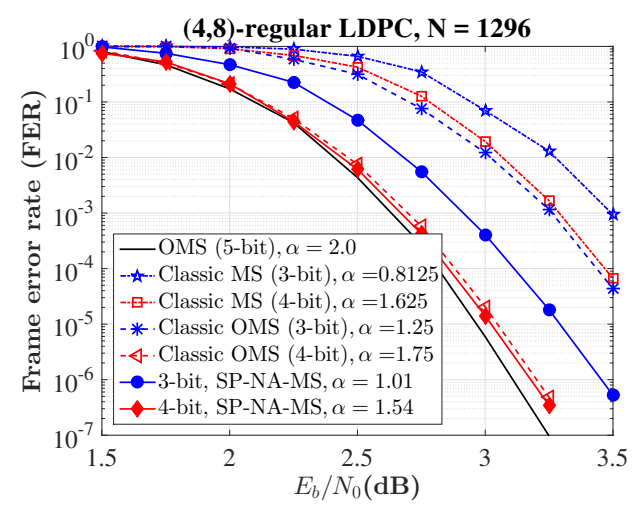

Figure 3: FER performance for $(4,8)$-regular LDPC code.

(i.e. at FER $=10^{-2}$ ) an SNR gain for the SP-NA-MS decoders which corresponds to the threshold differences: around 0.27 $\mathrm{dB}$ for $\left(q=3, d_{v}=3, R=1 / 2\right), 0.06 \mathrm{~dB}$ for $\left(q=4, d_{v}=\right.$ $3, R=1 / 2), 0.32 \mathrm{~dB}$ for $\left(q=3, d_{v}=4, R=1 / 2\right)$, and the same performance for $\left(q=4, d_{v}=4, R=1 / 2\right)$. We have made the same analysis for QC-LDPC codes with rate $R=3 / 4$, i.e. $\left(d_{v}=3, d_{c}=12\right)$ and $\left(d_{v}=4, d_{c}=16\right)$, and obtained that the SNR gains correspond to the DE gains.

Simulation results for the IEEE 802.3 ETHERNET code are provided on Fig. 4 with a maximum of 30 iterations. Again, the SNR gains in the waterfall correspond to what was predicted with the DE analysis, with a $0.19 \mathrm{~dB}$ gain for $q=3$ and the same performance for $q=4$.

\section{CONCLUSION}

In this paper we have proposed to use a sign-preserving factor which helps the message passing decoders to keep the sign information of extrinsic messages during the VNU processing. We have also proposed a noise model to introduce randomness in the message passing decoders. Both the signpreserving factor and the noise model help to improve the error correction performance of quantized iterative decoders. Density Evolution was used to obtain the optimum noise parameters and the optimum channel gain factor. The DE thresholds results have shown that the preservation of the sign of messages and the effect of the injected noise is

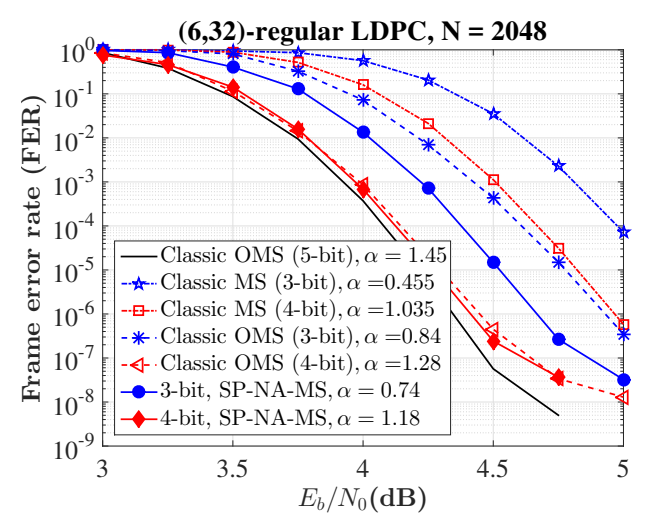

Figure 4: FER performance for the ETHERNET code.

always beneficial for low precision SP-NA-MS decoders. The finite-length Monte Carlo simulations have corroborated the DE thresholds. An efficient implementation of SP-NA-MS decoders can be made without noise injection since the noise parameters are close or equal to 0 or 1 .

\section{ACKNOWLEDGEMENT}

This work has been funded by the french ANR under grant number ANR-15-CE25-0006-01 (NAND project).

\section{REFERENCES}

[1] "IEEE Standard for Ethernet," IEEE Std 802.3-2015 (Revision of IEEE Std 802.3-2012), pp. 1-4017, March 2016.

[2] M. R. Tanner, "A Recursive Approach to Low Complexity Codes," IEEE Transactions on Information Theory, vol. Vol-27, no. 5, pp. 533-547, September 1981.

[3] J. Chen and M. P. C. Fossorier, "Density Evolution for Two Improved BP-Based Decoding Algorithms of LDPC Codes," IEEE Communications Letters, vol. Vol-6, no. 5, pp. 208-210, May 2002.

[4] J. Chen, A. Dholakia, E. Eleftheriou, M. P. C. Fossorier, and X.-Y. Hu, "Reduced-Complexity Decoding of LDPC Codes," IEEE Transactions on Communications, vol. Vol-53, no. 8, pp. 1288-1299, August 2005.

[5] L. Ping and W. K. Leung, "Decoding Low Density Parity Check Codes with Finite Quantization Bits," IEEE Communications Letters, vol. Vol4, no. 2, pp. 62-64, February 2000.

[6] J. Zhao, F. Zarkeshvari, and A. H. Banihashemi, "On Implementation of Min-Sum Algorithm and Its Modifications for Decoding Low-Density Parity-Check (LDPC) Codes," IEEE Transactions on Communications, vol. Vol-53, no. 4, pp. 549-554, April 2005.

[7] T. T. Nguyen-Ly, V. Savin, K. Le, D. Declercq, F. Ghaffari, and O. Boncalo, "Analysis and Design of Cost-Effective, High-Throughput LDPC Decoders," IEEE Transactions on Very Large Scale Integration (VLSI) Systems, vol. Vol. 26, no. 3, pp. 508-521, March 2018.

[8] F. Cochachin, D. Declercq, E. Boutillon, and L. Kessal, "Density Evolution Thresholds for Noise-Against-Noise Min-Sum Decoders," 2017 IEEE 28th Annual International Symposium on Personal, Indoor, and Mobile Radio Communications (PIMRC), pp. 1-7, Oct 2017.

[9] T. J. Richardson and R. L. Urbanke, "The Capacity of LowDensity Parity-Check Codes Under Message-Passing Decoding," IEEE Transactions on Information Theory, vol. Vol-47, no. 2, pp. 599-618, February 2001.

[10] L. R. Varshney, "Performance of LDPC Codes Under Noisy MessagePassing Decoding," ITW, pp. 178-183, September 2007.

[11] C. K. Ngassa, V. Savin, E. Dupraz, and D. Declercq, "Density evolution and functional threshold for the noisy min-sum decoder," IEEE Transactions on Communications, vol. Vol-63, no. 5, pp. 1497-1509, May 2015.

[12] A. Venkiah, D. Declercq, and C. Poulliat, "Design of Cages with a Randomized Progressive Edge Growth Algorithm," IEEE Communications Letters, vol. Vol. 12, no. 4, pp. 301-303, April 2008. 